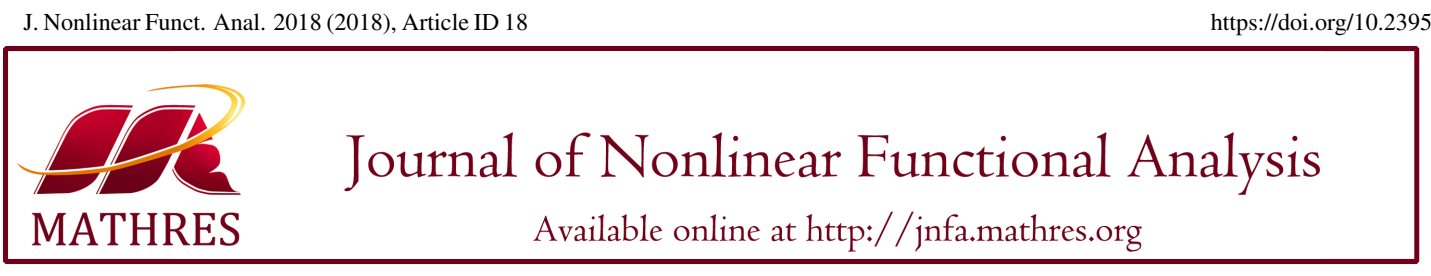

https://doi.org/10.23952/jnfa.2018.18

\title{
OPTIMALITY CONDITIONS FOR HENIG EFFICIENT AND SUPEREFFICIENT SOLUTIONS OF VECTOR EQUILIBRIUM PROBLEMS
}

\author{
DO VAN LUU ${ }^{1,2, *}$, TRAN THI MAI ${ }^{3}$ \\ ${ }^{1}$ TIMAS, Thang Long University, Hanoi, Vietnam \\ ${ }^{2}$ Institute of Mathematics, Vietnam Academy of Science and Technology, Hanoi, Vietnam \\ ${ }^{3}$ Thai Nguyen University of Economics and Business Administration, Vietnam
}

\begin{abstract}
Kuhn-Tucker necessary conditions for local Henig efficient and superefficient solutions of vector equilibrium problems involving equality, inequality and set constraints with locally Lipschitz functions are derived under the constraint qualification of Abadie type via the Michel-Penot subdifferentials. Under assumptions on the generalized convexity, Kuhn-Tucker necessary conditions for Henig efficiency and superefficiency become sufficient optimality conditions. Some applications to vector variational inequality and vector optimization problems are also given.
\end{abstract}

Keywords. Local Henig efficient solution; Local superefficient solution; Vector equilibrium problem; Vector variational inequality; Vector optimization problem.

2010 Mathematics Subject Classification. 90C46, 91B50, 49J52.

\section{INTRODUCTION}

The vector equilibrium problem provides an unified mathematical model which includes many other problems as special cases such as vector variational inequalities, vector optimization problems, vector saddle point problems, fixed point problems, vector complementarity problems, vector Nash equilibrium problems. It can be seen that the applied fields of vector equilibrium problems are greatly extensive. Optimality conditions for weakly efficient solutions, efficient solutions, Henig efficient solutions, globally efficient solutions and superefficient solutions of vector equilibrium problems have been studied by many authors; see, e.g., $[1,2,3,4,5,6,7,8,9,10,11,12,13]$ and references therein. There are a lot of work$\mathrm{s}$ to dealt with the existence and optimality conditions for Henig efficient solutions and superefficient solutions of vector equilibrium problems. Gong [3] derived optimality conditions for Henig efficient solutions and superefficient solutions of vector equilibrium problems with a set constraint. Long-HuangPeng [8] established optimality conditions for Henig efficient solutions and superefficient solutions of

${ }^{*}$ Corresponding author.

E-mail addresses: dvluu@ math.ac.vn (D.V. Luu), tranthimai879@ gmail.com (T.T. Mai).

Received December 12, 2017; Accepted May 1, 2018.

(C)2018 Journal of Nonlinear Functional Analysis 
vector equilibrium problems involving a cone-constraint and a set constraint with subconvexlike functions. Khanh-Tung [7] derived optimality conditions for various efficient solutions of constrained vector equilibrium problems using approximations. Ha [6] established optimality conditions for various efficient solutions of set-valued equilibrium problems via coderivatives. Recently, Luu-Hang [11] derived optimality conditions for efficient solutions of vector equilibrium problems involving equality and inequality constraints with locally Lipschitz functions in terms of the Clarke subdifferentials on using the notion of quasirelative interior of a convex set in an infinite dimensional space, but not for Henig efficient and superefficient solutions. Luu-Hang [12] established necessary conditions of Fritz John and Kuhn-Tucker types for weak efficient solutions of a nonsmooth vector equilibrium problem with equilibrium constraints via the Clarke subdifferentials. Making use of results on optimality conditions for nonsmooth optimization problems in terms of the Michel-Penot subdifferentials, we can derive optimality conditions for local Henig efficient solutions and local superefficient solutions of constrained vector equilibrium problems.

Motivated by the works $[3,8,11]$, in this paper we establish Kuhn-Tucker necessary conditions for local Henig efficient solutions and local superefficient solutions of vector equilibrium problems involving equality, inequality and set constraints with locally Lipschitz functions under the constraint qualification of Abadie type via the Michel-Penot subdifferentials. Under assumptions on generalized convexity, Kuhn-Tucker necessary conditions for Henig efficiency and superefficiency become sufficient optimality conditions.

The rest of the paper is organized as follows. In Section 2, some preliminaries are presented. In Section 3, based on a Kuhn-Tucker necessary condition by Luu [14] and a scalarization result by Gong [3], we derive Kuhn-Tucker necessary conditions for local Henig efficient solutions and local superefficient solutions of vector equilibrium problems involving equality, inequality and set constraints with locally Lipschitz functions under the constraint qualification of Abadie type via the Michel-Penot subdifferentials. The Kuhn-Tucker necessary conditions via the Michel-Penot subdifferentials can be sharper than those expressed in terms of the Clarke subdifferentials. This is shown in Examples 2.1 and 2.2 below. In Section 4, under assumptions on $\partial^{M P}$-pseudoconvexity and $\partial^{M P}$-quasiconvexity, Kuhn-Tucker necessary conditions for Henig efficiency and superefficiency become sufficient optimality conditions. Section 5 is devoted to discussion of applications to vector variational inequalities and vector optimization problems. Observe that the results obtained in this paper are more general than those obtained by Gong [3] for vector equilibrium problems with only a set constraint, and those obtained by Long-Huang-Peng [8] for vector equilibrium problems with subconvexlike functions.

\section{PRELIMINARIES}

Let $X$ be a Banach space and let $X^{*}$ be the topological dual of $X$. Let $\bar{x} \in X$ and let $f$ be a real-valued function defined on $X$. Following [15], the Michel-Penot directional derivative of $f$ at $\bar{X}$ in a direction $v \in X$ is defined as follows

$$
f^{\diamond}(\bar{x} ; v):=\sup _{w \in X} \limsup _{t \downarrow 0} \frac{f(\bar{x}+t(v+w))-f(\bar{x}+t w)}{t} .
$$

The Michel-Penot subdifferential of $f$ at $\bar{x}$ is

$$
\partial^{M P} f(\bar{x}):=\left\{\xi \in X^{*}:\langle\xi, v\rangle \leqslant f^{\diamond}(\bar{x} ; v), \forall v \in X\right\},
$$


where $\langle.,$.$\rangle denotes the coupling between X^{*}$ and $X$. Note that in case $f$ is Gâteaux differentiable at $\bar{x}$, one has (see [15]) $\partial^{M P} f(\bar{x})=\left\{\nabla_{G} f(\bar{x})\right\}$, where $\nabla_{G} f(\bar{x})$ denotes the Gâteaux derivative of $f$ at $\bar{x}$. Thus if $f$ is Fréchet differentiable at $\bar{x}$ with Fréchet derivative $\nabla f(\bar{x})$, then $\partial^{M P} f(\bar{x})=\{\nabla f(\bar{x})\}$.

For a function $f$ which is locally Lipschitz at $\bar{x}$ with constant $L$, the function $f^{\diamond}(\bar{x}$; .) is positively homogeneous, subadditive on $X$, Lipschitz with constant $L$ on $X, \partial^{M P} f(\bar{x})$ is a nonempty, convex, weak* compact subsets of $X^{*}$ and $\|\xi\| \leqslant L$ for all $\xi \in \partial^{M P} f(\bar{x})$. Moreover, for all $v \in X$,

$$
f^{\diamond}(\bar{x} ; v)=\max \left\{\langle\xi, v\rangle: \xi \in \partial^{M P} f(\bar{x})\right\} .
$$

Following [16], the Clarke generalized directional derivative of $f$ at $\bar{x}$, with respect to a direction $v$, is defined as

$$
f^{0}(\bar{x} ; v)=\limsup _{x \rightarrow \bar{x}, t \downarrow 0} \frac{f(x+t v)-f(x)}{t} .
$$

The Clarke subdifferential of $f$ at $\bar{x}$ is

$$
\partial^{C} f(\bar{x})=\left\{\xi \in X^{*}:\langle\xi, v\rangle \leqslant f^{0}(\bar{x} ; v), \forall v \in X\right\} .
$$

It is obvious that for a function $f$ which is locally Lipschitz at $\bar{x}$,

$$
\begin{aligned}
& f^{\diamond}(\bar{x} ; v) \leqslant f^{0}(\bar{x} ; v) \quad(\forall v \in X), \\
& \partial^{M P} f(\bar{x}) \subseteq \partial^{C} f(\bar{x}) .
\end{aligned}
$$

(see [17], Proposition 7.3.7).

If $f: \mathbb{R}^{n} \rightarrow \mathbb{R}^{p}$ is a locally Lipschitz mapping at $\bar{x}$, the Clarke generalized Jacobian of $f$ at $\bar{x}$ is defined as (see [16]):

$$
\partial_{J} f(\bar{x})=\operatorname{co}\left\{\lim _{i \rightarrow+\infty} \nabla f\left(x_{i}\right): x_{i} \rightarrow \bar{x}, x_{i} \in S\right\},
$$

where $\nabla f\left(x_{i}\right)$ denotes the Jacobian of $f$ at $x_{i}$, co indicates the convex hull, and $S$ stands for the set of points for which $f$ is differentiable. In case $n=1$, the Clarke generalized Jacobian of $f$ at $\bar{x}$ coincides with the Clarke subfifferential of $f$ at $\bar{x}$.

The following examples show that the subdifferentials $\partial^{M P} f(\bar{x})$ and $\partial^{C} f(\bar{x})$ may be greatly different.

Example 2.1. The function $f$ be defined on $\mathbb{R}$ as

$$
f(x)= \begin{cases}x^{2} \sin \frac{1}{x}, & x \neq 0, \\ 0, & x=0 .\end{cases}
$$

For $\bar{x}=0, \partial^{M P} f(0)=\{0\}, f^{\diamond}(0 ; v)=0(\forall v \in \mathbb{R})$. By Example 2.2.3 in [16], $f^{0}(0 ; v)=|v|, \partial^{C} f(0)=$ $[-1,1]$, and thus, $\partial^{M P} f(0) \varsubsetneqq \partial^{C} f(0)$.

Example 2.2. The function $f$ be defined on $\mathbb{R}$ as

$$
f(x)= \begin{cases}x^{2}\left|\cos \frac{\pi}{x}\right|, & x \neq 0, \\ 0, & x=0 .\end{cases}
$$

Then, $\partial^{M P} f(0)=\{0\}, f^{\diamond}(0 ; v)=0(\forall v \in \mathbb{R}), f^{0}(0 ; v)=\pi|v|(\forall v \in \mathbb{R}), \partial^{C} f(0)=[-\pi, \pi]$. Thus, $\partial^{M P} f(0) \varsubsetneqq \partial^{C} f(0)$. 
If $f$ is a convex function on $X$, then the subdifferential of $f$ at $\bar{x}$ is defined as

$$
\partial f(\bar{x}):=\left\{\xi \in X^{*}:\langle\xi, x-\bar{x}\rangle \leqslant f(x)-f(\bar{x}), \forall x \in X\right\} .
$$

Proposition 7.3.9 [17] was pointed that if $f$ is convex on $X$ and locally Lipschitz at $\bar{x} \in X$, then

$$
\partial^{M P} f(\bar{x})=\partial f(\bar{x})=\partial^{C} f(\bar{x}) .
$$

Let $f$ be a mapping from $X$ into another Banach space $Y$. The mapping $f$ is called strictly diifferentiable at $\bar{x} \in X$ if there is a continuous linear mapping $D_{s} f(\bar{x}): X \rightarrow Y$ such that for each $v \in X$,

$$
D_{s} f(\bar{x}) v=\lim _{x \rightarrow \bar{x}, t \downarrow 0} \frac{f(x+t v)-f(x)}{t}
$$

holds, and the convergence is uniform with respect to $v$ in compact sets.

Recall [16] that the Clarke tangent cone to a nonempty closed set $C \subseteq X$ at a point $\bar{x} \in C$ is defined as

$$
\begin{aligned}
& T(C ; \bar{x}):=\left\{v \in X: \forall x_{n} \in C, x_{n} \rightarrow \bar{x}, \forall t_{n} \downarrow 0, \exists v_{n} \rightarrow v\right. \\
&\text { such that } \left.x_{n}+t_{n} v_{n} \in C, \forall n\right\} .
\end{aligned}
$$

The Clarke normal cone to $C$ at $\bar{x}$ is

$$
N(C ; \bar{x}):=\left\{\xi \in X^{*}:\langle\xi, v\rangle \leqslant 0, \forall v \in T(C ; \bar{x})\right\} .
$$

Thus $N(C ; \bar{x})=-T(C ; \bar{x})^{*}$, where $T(C ; \bar{x})^{*}$ is the dual cone of $T(C ; \bar{x})$. Note that the cones $T(C ; \bar{x})$ and $N(C ; \bar{x})$ are nonempty convex, $T(C ; \bar{x})$ is closed and $N(C ; \bar{x})$ is weakly* closed.

\section{KUHN-TUCKER NECESSARY CONDITIONS FOR LOCAL HENIG EFFICIENT AND SUPEREFFICIENT \\ SOLUTIONS}

This section deals with Kuhn-Tucker necessary conditions for local Henig efficient and superefficient solutions of vector equilibrium problems via the Michel-Penot subdifferentials. The Kuhn-Tucker necessary conditions obtained here via the Michel-Penot subdifferentials may be sharper than those expressed in terms of the Clarke subdifferentials. This has been shown in Examples 2.1 and 2.2.

Let $X$ be a real Banach space and let $X^{*}$ be the topological dual of $X$. Let $K$ be a nonempty closed subset of $X$. Let $F$ be a mapping from $K \times K$ to another Banach space $Y$ and $Q$ a pointed closed convex cone in $Y$. Let us consider the following vector equilibrium problem (VEP): Finding a point $x \in K$ such that

$$
F(x, y) \notin-Q \backslash\{0\}(\forall y \in K) .
$$

Definition 3.1. A vector $\bar{x}$ solved (3.1) will be called an efficient solution of (VEP).

Denote the dual cone of $Q$ by $Q^{*}:=\left\{y^{*} \in Y^{*}:\left\langle y^{*}, y\right\rangle \geqslant 0, \forall y \in Q\right\}$. A nonempty convex subset $B$ of $Q$ is called a base of $Q$, if $Q=\operatorname{cone} B$ and $0 \notin \mathrm{cl} B$, where cl stands for the closure, cone $B$ denotes the cone hull of $B: \operatorname{cone} B=\{t b: t \geqslant 0, b \in B\}$. Denote the quasi-interior of $Q^{*}$ by $Q^{\#}:=\left\{y^{*} \in Y^{*}:\left\langle y^{*}, y\right\rangle>\right.$ $0, \forall y \in Q \backslash\{0\}\}$. We set $Q^{\Delta}(B):=\left\{y^{*} \in Q^{\#}: \exists t>0\right.$ such that $\left.\left\langle y^{*}, b\right\rangle \geqslant t, \forall b \in B\right\}$. If $B$ is a base of the cone $Q$, by the separation theorem 3.6 [5], there exists $y^{*} \in Y^{*} \backslash\{0\}$ such that

$$
\alpha:=\inf \left\{\left\langle y^{*}, b\right\rangle: b \in B\right\}>y^{*}(0)=0 .
$$


Then the set $V_{B}:=\left\{y \in Y:\left|\left\langle y^{*}, y\right\rangle\right|<\frac{\alpha}{2}\right\}$ is an absolutely convex open neighborhood of $0 \in Y$ (see [4]), and $\inf \left\{\left\langle y^{*}, y\right\rangle: y \in B+V_{B}\right\} \geqslant \frac{\alpha}{2}$. For each convex neighborhood $U$ of $0, U \subseteq V_{B}$, one has $0 \notin \operatorname{cl}(B+U)$. Hence, the set $Q_{U}(B):=\operatorname{cone}(U+B)$ is a pointed convex cone, and

$$
Q \backslash\{0\} \subseteq \operatorname{int} Q_{U} B
$$

The following result is needed in the sequel.

Proposition 3.2. [3] If the pointed closed convex cone $Q$ has a base $B$, then

(a) For any open absolutely convex neighborhood $U$ of $0, U \subset V_{B}$, one has

$$
Q_{U}(B)^{*} \backslash\{0\} \subseteq Q^{\Delta}(B) .
$$

(b) If $B$ is bounded and closed, then int $Q^{*}=Q^{\Delta}(B)$, where int $Q^{*}$ is the interior of $Q^{*}$ in $Y^{*}$ with respect to the strong topology of $Y^{*}$.

Definition 3.3. A vector $\bar{x} \in K$ is called a Henig efficient solution of (VEP) if there is an absolutely convex neighborhood $U$ of $0, U \subseteq V_{B}$ such that

$$
\operatorname{cone} F(\bar{x}, K) \cap\left(-\operatorname{int} Q_{U}(B)\right)=\emptyset,
$$

where $F(\bar{x}, K)=\bigcup_{y \in K} F(\bar{x}, y)$. Since $Q_{U}(B)$ is a pointed convex cone, $\bar{x}$ is a Henig solution if and only if

$$
F(\bar{x}, K) \cap\left(-\operatorname{int} Q_{U}(B)\right)=\emptyset .
$$

Note that $\bar{x} \in K$ is a Henig efficient solution of (VEP) if and only if there is an absolutely convex neighborhood $U$ of $0, U \subseteq V_{B}$ such that (see [3])

$$
\text { cone } F(\bar{x}, K) \cap(U-B)=\emptyset \text {. }
$$

Definition 3.4. A vector $\bar{x} \in K$ is called a superefficient solution of (VEP) if for each neighborhood $V$ of 0 , there is a neighborhood $U$ of 0 such that

$$
\operatorname{cone} F(\bar{x}, K) \cap(U-Q) \subseteq V
$$

In Definitions 3.1, 3.3 and 3.4, replacing $K$ by $K \cap W$ for some neighborhood $W$ of $\bar{x}$, we obtain the notions of local efficient solution, local Henig efficient solution and local superefficient solution for (VEP), respectively.

Remark 3.5. It follows from (3.2) and (3.3) that a Henig efficient (local Henig efficient) solution is also an efficient (resp. local efficient) solution.

We recall the following result which provides a relationship between Henig efficient solutions and superfficient solutions.

Proposition 3.6. [4] Let $B$ be a base of $Q$.

(a) If $\bar{x} \in K$ is a superefficient solution to (VEP), then it is also a Henig efficient solution.

(b) If $B$ is bounded and closed, then a Henig efficient solution to (VEP) is a superefficient solution as well. 
Let $g$ and $h$ be mappings from $X$ into $\mathbb{R}^{m}$ and $\mathbb{R}^{\ell}$, respectively, and let $C$ be nonempty closed subsets of $X$. Then $g$ and $h$ can be expressed as follows: $g=\left(g_{1}, \ldots, g_{m}\right), h=\left(h_{1}, \ldots, h_{\ell}\right)$. This paper deals with the vector equilibrium problem (VEP) in which $K$ is described by

$$
K=\{x \in C: g(x) \leqslant 0, h(x)=0\} .
$$

This constrained vector equilibrium problem is denoted by (CVEP). For the sake of simplicity, we set $I:=\{1, \ldots, m\}$ and $L:=\{1, \ldots, \ell\}$. For $\bar{x} \in K$, we denote

$$
F_{\bar{x}}(y):=F(\bar{x}, y), I(\bar{x}):=\left\{i \in I: g_{i}(\bar{x})=0\right\} .
$$

The following assumption is posed on Problem (CVEP):

Assumption 3.7. The functions $F_{\bar{x}}, g_{i}(i \in I(\bar{x}))$ are locally Lipschitz at $\bar{x}, h_{j}(j \in L)$ are Fréchet differentiable at $\bar{x}$ and $Q$ has a base $B$.

Let us consider the following sets:

$$
\begin{aligned}
C(K ; \bar{x}):=\left\{v \in T(C ; \bar{x}): g_{i}^{\diamond}(\bar{x}, v) \leqslant 0(\forall i \in I(\bar{x})),\right. \\
\left.\left\langle\nabla h_{j}(\bar{x}), v\right\rangle=0(j=1, \ldots, \ell)\right\}, \\
H(\bar{x}):=\bigcup\left\{\sum_{i \in I(\bar{x})} \mu_{i} \partial^{M P} g_{i}(\bar{x})+\sum_{j=1}^{\ell} v_{j} \nabla h_{j}(\bar{x})\right. \\
\left.+N(C ; \bar{x}): \mu_{i} \geqslant 0(\forall i \in I(\bar{x})), v_{j} \in \mathbb{R}(j=1, \ldots, \ell)\right\} .
\end{aligned}
$$

To derive Kuhn-Tucker necessary conditions for Problem (CVEP), we introduce the following constraint qualification (CQ):

$$
C(K ; \bar{x}) \subseteq T(K ; \bar{x}) .
$$

The constraint qualification of this type was studied in [10, 14].

We shall begin with establishing a necessary optimality condition for local Henig efficient solution of (CVEP).

Theorem 3.8. Let $\bar{x}$ be a local Henig efficient solution of $(C V E P)$. Assume that $F_{\bar{x}}(\bar{x})=0, H(\bar{x})$ is weakly ${ }^{*}$ closed, Assumption 3.7 and Constraint qualification $(C Q)$ hold. Then there exist $\bar{\mu}_{i} \geqslant 0(i \in I(\bar{x}))$, $\bar{v}_{j} \in \mathbb{R}(j \in J)$, and a continuous positively homogeneous function $\Lambda$ on $Y$ satisfying

( $\alpha)$ If $y_{2}-y_{1} \in Q \backslash\{0\}$, then $\Lambda\left(y_{1}\right)<\Lambda\left(y_{2}\right)$,

( $\beta) \exists \beta_{0}>0$ such that $\Lambda(-b) \leqslant-\beta_{0}(\forall b \in B)$,

such that

$$
0 \in \partial^{M P}\left(\Lambda \circ F_{\bar{x}}\right)(\bar{x})+\sum_{i \in I(\bar{x})} \bar{\mu}_{i} \partial^{M P} g_{i}(\bar{x})+\sum_{j \in L} \bar{v}_{j} \nabla h_{j}(\bar{x})+N_{C}(\bar{x})
$$

Proof. Since $\bar{x}$ is a local Henig efficient solution of (CVEP), we see that there are a neighborhood $W$ of $\bar{x}$ and an absolutely convex neighborhood $U$ of $0, U \subseteq V_{B}$ such that

$$
\operatorname{cone} F(\bar{x}, K \cap W) \cap\left(-\operatorname{int} Q_{U}(B)\right)=\emptyset .
$$


Applying Theorem 3.2 [3] yields the existence a continuous positively homogeneous subadditive function $\Lambda$ on $Y$ such that $(\alpha),(\beta)$ hold, and

$$
\left(\Lambda \circ F_{\bar{x}}\right)(x) \geqslant 0(\forall x \in K \cap W) .
$$

Since $F_{\bar{x}}(\bar{x})=0$ and $\Lambda$ is positively homogeneous, we have $\left(\Lambda \circ F_{\bar{x}}\right)(\bar{x})=0$. In view of (3.7), we deduce that $\bar{x}$ is a minimum of the following scalar optimization problem:

$$
\begin{array}{ll} 
& \min \left(\Lambda \circ F_{\bar{x}}\right)(x), \\
\text { s.t. } \quad & g_{i}(x) \leqslant 0(i \in I), \\
& h_{j}(x)=0(j \in J), \\
& x \in C \cap W .
\end{array}
$$

Since the function $\Lambda$ is continuous and convex, we can apply Proposition 2.2.6 [16] to deduce that it is locally Lipschitz. Moreover, it follows from (CQ) that

$$
\begin{aligned}
C(K \cap W ; \bar{x}) & =C(K ; \bar{x}) \\
& \subseteq T(K ; \bar{x})=T(K \cap W ; \bar{x}) .
\end{aligned}
$$

Thus the constraint qualification (CQ) holds for Problem (P). Taking account of Theorem 3.1 [14] to the scalar problem (P) yields the existence of $\bar{\mu}_{i} \geqslant 0(\forall i \in I(\bar{x})), \bar{v}_{j} \in \mathbb{R}(\forall j \in L)$ such that

$$
0 \in \partial^{M P}\left(\Lambda \circ F_{\bar{x}}\right)(\bar{x})+\sum_{i \in I(\bar{x})} \bar{\mu}_{i} \partial^{M P} g_{i}(\bar{x})+\sum_{j \in L} \bar{v}_{j} \nabla h_{j}(\bar{x})+N_{C \cap W}(\bar{x}) .
$$

On the other hand,

$$
N_{C \cap W}(\bar{x})=N_{C}(\bar{x}) .
$$

Hence, (3.8) implies (3.7). This completes the proof.

From Theorem 3.8, we obtain a necessary optimality condition for local superefficient solution of (CVEP).

Corollary 3.9. Let $\bar{x}$ be a local superefficient solution of $(C V E P)$. Assume that $F_{\bar{x}}(\bar{x})=0, H(\bar{x})$ is weakly ${ }^{*}$ closed, Assumption 3.7 and Constraint qualification $(C Q)$ hold. Then there exist $\bar{\mu}_{i} \geqslant 0(i \in I(\bar{x}))$, $\bar{v}_{j} \in \mathbb{R}(j \in J)$, and a continuous positively homogeneous function $\Lambda$ on $Y$ satisfying $(\alpha)$, $(\beta)$ such that

$$
0 \in \partial^{M P}\left(\Lambda \circ F_{\bar{x}}\right)(\bar{x})+\sum_{i \in I(\bar{x})} \bar{\mu}_{i} \partial^{M P} g_{i}(\bar{x})+\sum_{j \in L} \bar{v}_{j} \nabla h_{j}(\bar{x})+N_{C}(\bar{x}) .
$$

Proof. Taking account of Proposition 3.6, we deduce that $\bar{x}$ is a local Henig efficient solution of (CVEP). By virtue of Theorem 3.8, we obtain the desired conclusion.

In case that $X$ and $Y$ are finite-dimensional, a Kuhn-Tucker necessary condition can be stated as follows.

Theorem 3.10. Let $X=\mathbb{R}^{n}, Y=\mathbb{R}^{p}$ and let $\bar{x}$ be a local Henig efficient solution of (CVEP). Assume that all the hypotheses of Theorem 3.8 hold. Then

(i) There exist $\bar{\lambda} \in Q^{\Delta}(B), \bar{\mu}_{i} \geqslant 0(i \in I(\bar{x})), \bar{v}_{j} \in \mathbb{R}(j \in J)$, such that

$$
0 \in \bar{\lambda} \partial_{J} F_{\bar{x}}(\bar{x})+\sum_{i \in I(\bar{x})} \bar{\mu}_{i} \partial^{M P} g_{i}(\bar{x})+\sum_{j \in L} \bar{v}_{j} \nabla h_{j}(\bar{x})+N_{C}(\bar{x}) .
$$


(ii) Moreover, if the base $B$ of $Q$ is bounded and closed, then $\bar{\lambda} \in$ int $Q^{*}$.

Proof. (i) We invoke Theorem 3.8 to deduce that there exist $\bar{\mu}_{i} \geqslant 0(i \in I(\bar{x})), \bar{v}_{j} \in \mathbb{R}(j \in J)$, and a continuous positively homogeneous function $\Lambda$ on $Y$ satisfying $(\alpha),(\beta)$ such that

$$
0 \in \partial^{M P}\left(\Lambda \circ F_{\bar{x}}\right)(\bar{x})+\sum_{i \in I(\bar{x})} \bar{\mu}_{i} \partial^{M P} g_{i}(\bar{x})+\sum_{j \in L} \bar{v}_{j} \nabla h_{j}(\bar{x})+N_{C}(\bar{x}) .
$$

Since the function $\Lambda$ is continuous and convex, we can apply Proposition 2.2.6 [16] to deduce that it is locally Lipschitz, and so is the function $\Lambda \circ F_{\bar{x}}$. Hence,

$$
\partial^{M P}\left(\Lambda \circ F_{\bar{x}}\right)(\bar{x}) \subseteq \partial^{C}\left(\Lambda \circ F_{\bar{x}}\right)(\bar{x}) .
$$

Taking account of Theorem 2.6.6 [16] on a Jacobian chain rule, we get

$$
\partial^{C}\left(\Lambda \circ F_{\bar{x}}\right)(\bar{x}) \subseteq \operatorname{co}\left\{\partial^{C} \Lambda\left(F_{\bar{x}}(\bar{x})\right) \partial_{J} F_{\bar{x}}(\bar{x})\right\} .
$$

Combining (3.10), (3.11) and (3.12) yields that there exist $\lambda_{i} \geqslant 0(i=1, \ldots, r), \sum_{i=1}^{r} \lambda_{i}=1, \xi_{i} \in \partial^{C} \Lambda\left(F_{\bar{x}}(\bar{x}), \eta_{i} \in\right.$ $\partial_{J} F_{\bar{x}}(\bar{x})$ such that

$$
\begin{aligned}
0 & \in \sum_{i=1}^{r} \lambda_{i} \xi_{i} \eta_{i}+\sum_{i \in I(\bar{x})} \bar{\mu}_{i} \partial^{M P} g_{i}(\bar{x})+\sum_{j \in L} \bar{v}_{j} \nabla h_{j}(\bar{x})+N_{C}(\bar{x}) \\
& \subseteq \sum_{i=1}^{r} \lambda_{i} \xi_{i} \partial_{J} F_{\bar{x}}(\bar{x})+\sum_{i \in I(\bar{x})} \bar{\mu}_{i} \partial^{M P} g_{i}(\bar{x})+\sum_{j \in L} \bar{v}_{j} \nabla h_{j}(\bar{x})+N_{C}(\bar{x}) .
\end{aligned}
$$

On the other hand, in view of the convexity of $\partial^{C} \Lambda\left(F_{\bar{x}}(\bar{x})\right)$, one gets $\bar{\lambda}:=\sum_{i=1}^{r} \lambda_{i} \xi_{i} \in \partial^{C} \Lambda\left(F_{\bar{x}}(\bar{x})\right)$. Hence, it follows from (3.13) that

$$
0 \in \bar{\lambda} \partial_{J} F_{\bar{x}}(\bar{x})+\sum_{i \in I(\bar{x})} \bar{\mu}_{i} \partial^{M P} g_{i}(\bar{x})+\sum_{j \in L} \bar{v}_{j} \nabla h_{j}(\bar{x})+N_{C}(\bar{x}) .
$$

Let us see that $\bar{\lambda} \in Q^{\Delta}(B)$. In fact, since the function $\Lambda$ is locally Lipschitz convex and $\bar{\lambda} \in \partial^{C} \Lambda\left(F_{\bar{x}}(\bar{x})\right)$, it follows from Theorem 3.2 [3] that there exists $\beta_{0}>0$ such that for every $y \in B$,

$$
\begin{aligned}
\langle\bar{\lambda},-y\rangle & =\left\langle\bar{\lambda}, F_{\bar{x}}(\bar{x})-y-F_{\bar{x}}(\bar{x})\right\rangle \\
& \leqslant \Lambda\left(F_{\bar{x}}(\bar{x})-y\right)-\Lambda\left(F_{\bar{x}}(\bar{x})\right)=\Lambda(-y)<-\beta_{0} .
\end{aligned}
$$

Hence, $\langle\bar{\lambda}, y\rangle>\beta_{0}(\forall y \in B)$, and so, $\bar{\lambda} \in Q^{\Delta}(B)$. The conclusion (3.9) follows.

(ii) Taking account of Proposition 3.2, we get

$$
Q^{\Delta}(B)=\operatorname{int} Q^{*} \text {. }
$$

Combining (3.14) and (3.15), we get the desired conclusion.

Corollary 3.11. Let $X=\mathbb{R}^{n}, Y=\mathbb{R}^{p}$ and $\bar{x}$ be a local superefficient solution of (CVEP). Assume that all the hypotheses of Theorem 3.8 hold. Then

(i) There exist $\bar{\lambda} \in Q^{\Delta}(B), \bar{\mu}_{i} \geqslant 0(i \in I(\bar{x})), \bar{v}_{j} \in \mathbb{R}(j \in J)$, such that

$$
0 \in \bar{\lambda} \partial_{J} F_{\bar{x}}(\bar{x})+\sum_{i \in I(\bar{x})} \bar{\mu}_{i} \partial^{M P} g_{i}(\bar{x})+\sum_{j \in L} \bar{v}_{j} \nabla h_{j}(\bar{x})+N_{C}(\bar{x}) .
$$

(ii) Moreover, if the base $B$ of $Q$ is bounded and closed, then $\bar{\lambda} \in$ int $Q^{*}$. 
Proof. Since $\bar{x}$ is a local superefficient solution of (CVEP), by Proposition 3.6, it is a local Henig efficient solution of (CVEP). Hence, all the hypotheses of Theorem 3.2 are fulfilled. Applying Theorem 3.10 yields the existence of $\bar{\lambda} \in Q^{\Delta}(B), \bar{\mu}_{i} \geqslant 0(i \in I(\bar{x})), \bar{v}_{j} \in \mathbb{R}(j \in J)$, such that (3.16) holds. If the base $B$ of $Q$ is a bounded closed set, then by Proposition 3.2, we have $Q^{\Delta}(B)=\operatorname{int} Q^{*}$. Hence, $\bar{\lambda} \in \operatorname{int} Q^{*}$, which completes the proof.

In case $X$ and $Y$ may be infinite-dimensional and the mapping $F_{\bar{x}}$ is strictly differentiable at $\bar{x}$ we get the following Kuhn-Tucker necessary condition for Henig efficiency.

Theorem 3.12. Let $\bar{x}$ be a local Henig efficient solution of (CVEP). Assume that all the hypotheses of Theorem 3.8 hold. Suppose, in addition, that $F_{\bar{x}}$ is strictly differentiable with the strict derivative $D_{s} F_{\bar{x}}(\bar{x})$. Then

(i) There exist $\bar{\lambda} \in Q^{\Delta}(B), \bar{\mu}_{i} \geqslant 0(i \in I(\bar{x})), \bar{v}_{j} \in \mathbb{R}(j \in J)$, such that

$$
0 \in\left[D_{s} F_{\bar{x}}(\bar{x})\right]^{*} \bar{\lambda}+\sum_{i \in I(\bar{x})} \bar{\mu}_{i} \partial^{M P} g_{i}(\bar{x})+\sum_{j \in L} \bar{v}_{j} \nabla h_{j}(\bar{x})+N_{C}(\bar{x})
$$

(ii) Moreover, if the base $B$ of $Q$ is bounded and closed, then $\bar{\lambda} \in$ int $Q^{*}$, where int $Q^{*}$ is the interior of $Q^{*}$ with respect to the strong topology on $Y^{*}$.

Proof. As also in the proof of Theorem 3.10, we invoke Theorem 3.8 to deduce that there exist $\bar{\mu}_{i} \geqslant$ $0(i \in I(\bar{x})), \bar{v}_{j} \in \mathbb{R}(j \in J)$, and a continuous positively homogeneous function $\Lambda$ on $Y$ satisfying $(\alpha)$, $(\beta)$ such that (3.10) holds. Since the function $\Lambda$ is continuous convex, we can apply Proposition 2.2.6 [16] to deduce that it is locally Lipschitz, and so is the function $\Lambda \circ F_{\bar{x}}$. Hence, (3.11) holds. Taking account of Theorem 2.3.10 [16], we get

$$
\partial^{C}\left(\Lambda \circ F_{\bar{x}}\right)(\bar{x}) \subseteq\left[D_{s} F_{\bar{x}}(\bar{x})\right]^{*} \partial^{C} \Lambda\left(F_{\bar{x}}(\bar{x})\right) .
$$

It follows that there exists $\bar{\lambda} \in \partial^{C} \Lambda\left(F_{\bar{x}}(\bar{x})\right)$ such that (3.17) holds. The remainder is analogously proved as Theorem 3.10.

From Theorem 3.12, we obtain the following Kuhn-Tucker necessary condition for superefficiency.

Corollary 3.13. Let $\bar{x}$ be a local superefficient efficient solution of $(C V E P)$. Assume that all the hypotheses of Theorem 3.8 hold. Suppose, in addition, that $F_{\bar{x}}$ is strictly differentiable with the strict derivative $D_{s} F_{\bar{x}}(\bar{x})$. Then

(i) There exist $\bar{\lambda} \in Q^{\Delta}(B), \bar{\mu}_{i} \geqslant 0(i \in I(\bar{x})), \bar{v}_{j} \in \mathbb{R}(j \in J)$, such that

$$
0 \in\left[D_{s} F_{\bar{x}}(\bar{x})\right]^{*} \bar{\lambda}+\sum_{i \in I(\bar{x})} \bar{\mu}_{i} \partial^{M P} g_{i}(\bar{x})+\sum_{j \in L} \bar{v}_{j} \nabla h_{j}(\bar{x})+N_{C}(\bar{x}) .
$$

(ii) If, moreover, the base B of $Q$ is bounded and closed, then $\bar{\lambda} \in$ int $Q^{*}$, where int $Q^{*}$ is the interior of $Q^{*}$ with respect to the strong topology on $Y^{*}$.

Proof. Applying Proposition 3.6 and Theorem 3.12, we get the desired conclusion.

Theorem 3.8 can be illustrated by the following example. 
Example 3.14. Let $X=\mathbb{R}^{2}, Y=\mathbb{R}^{3}, C=[0,1] \times[0,1], \bar{x}=(0,0), Q=\mathbb{R}_{+}^{3}$. Let $F: \mathbb{R}^{2} \times \mathbb{R}^{2} \rightarrow \mathbb{R}^{3}, g$ : $\mathbb{R}^{2} \rightarrow \mathbb{R}^{2}$ be defined as

$$
\begin{gathered}
F(x, y)=\left(\frac{1}{2} y_{2}^{2}\left(1-\tan x_{1}\right),\left|y_{2}\right|+y_{1}^{3}-y_{1} \operatorname{sgn} x_{2}, y_{1}\left(\sin x_{2}-\frac{1}{2}\right)\right), \\
g=\left(g_{1}, g_{2}\right), \\
g_{1}(y)=e^{y_{1}}-4, \\
g_{2}(y)=\left|y_{1}\right|-\left|y_{2}\right| \\
\left(x=\left(x_{1}, x_{2}\right) \in \mathbb{R}^{2}, y=\left(y_{1}, y_{2}\right) \in \mathbb{R}^{2}\right) . \text { Then }
\end{gathered}
$$

$$
F_{\bar{x}}(y)=\left(\frac{1}{2} y_{2}^{2},\left|y_{2}\right|+y_{1}^{3},-\frac{1}{2} y_{1}\right) .
$$

We have $K=\left\{\left(y_{1}, y_{2}\right) \in[0,1] \times[0,1]: y_{1} \leqslant y_{2}\right\}, T(C ; \bar{x})=\mathbb{R}_{+}^{2}$, and $N(C ; \bar{x})=\mathbb{R}_{-}^{2}$, where $\mathbb{R}_{-}=-\mathbb{R}_{+}$. Hence, $T(K ; \bar{x})=\left\{\left(y_{1}, y_{2}\right) \in \mathbb{R}_{+}^{2}: y_{1} \leqslant y_{2}\right\}, N(K ; \bar{x})=\mathbb{R}_{-}^{2} \cup\left\{\left(y_{1}, y_{2}\right) \in \mathbb{R}^{2}: 0 \geqslant-y_{2} \geqslant y_{1}\right\}$.

The pointed closed cone $Q$ has the following bounded closed convex base

$$
B=\left\{\left(y_{1}, y_{2}, y_{3}\right) \in \mathbb{R}_{+}^{3}: y_{1}+y_{2}+y_{3}=1\right\} .
$$

It is easy to check that $\operatorname{dist}(0, B)=\frac{\sqrt{3}}{3}$, where $\operatorname{dist}(0, B)$ indicates the distance from 0 to $B$. Taking $U$ being the open ball of radius $\delta=\frac{1}{4}$ around 0 , we see that $U$ is an absolutely convex neighborhood of 0 in $\mathbb{R}^{3}$, and

$$
\text { cone } F(\bar{x}, K) \cap(U-B)=\emptyset .
$$

Hence, $\bar{x}=0$ is a Henig efficient solution of the following vector equilibrium problem: Finding $x \in K$ such that

$$
F(x, y) \notin-Q \backslash\{0\}(\forall y \in K) .
$$

It can be seen that $\partial^{M P} g_{1}(\bar{x})=\{(1,0)\}$. By Example 2.1 [18],

$$
\begin{aligned}
\partial^{M P} g_{2}(\bar{x}) & =\operatorname{co}\{(1,1),(-1,1),((1,-1),(-1,-1)\} \\
& =\{(\eta, \zeta):-1 \leqslant \eta \leqslant 1,-1 \leqslant \zeta \leqslant 1\},
\end{aligned}
$$

where co indicates the convex hull. Hence, $g_{1}^{\diamond}(\bar{x} ;(u, v))=u, g_{2}^{\diamond}(\bar{x} ;(u, v))=|u|+|v|$, and so, $C(K ; \bar{x})=$ $\{(0,0)\}$. Therefore, $C(K ; \bar{x}) \subseteq T(K ; \bar{x})$. It can be seen that $H(\bar{x})$ is closed. Thus all the hypotheses of Theorem 3.8 are fulfilled. Taking account of Proposition 3.2, we get $Q^{\Delta}(B)=\operatorname{int} Q^{*}$, as $Q^{*}=\mathbb{R}_{+}^{3}$. Taking $\Lambda=(1,1,1) \in \operatorname{int} Q^{*}$, it has the properties in Proposition 3.2. Then

$$
\begin{gathered}
\left(\Lambda \circ F_{\bar{x}}\right)(y)=\frac{1}{2} y_{2}^{2}+\left|y_{2}\right|+y_{1}^{3}-\frac{1}{2} y_{1}, \\
\partial^{M P}\left(\Lambda \circ F_{\bar{x}}\right)(\bar{x})=\left\{\left(-\frac{1}{2}, \xi\right)^{T}:-1 \leqslant \xi \leqslant 1\right\},
\end{gathered}
$$

where the superscript ${ }^{T}$ denotes transposition. For $\mu=(1,1)$, the optimality condition (3.6) in Theorem 3.8 holds at $\bar{x}=(0,0)$ :

$$
(0,0)^{T} \in\left(\begin{array}{ll}
-\frac{1}{2} & \xi
\end{array}\right)^{T}+\left(\begin{array}{ll}
1 & 1
\end{array}\right)\left(\begin{array}{cc}
1 & 0 \\
\eta & \zeta
\end{array}\right)+\mathbb{R}_{-}^{2},
$$

for $\xi=\eta=-\frac{1}{2}, \zeta=\frac{1}{2}$. 


\section{SUFFICIENT CONDITIONS FOR HENIG EFFICIENT AND SUPEREFFICIENT SOLUTIONS}

In order to derive sufficient conditions for Henig efficient and superefficient solutions of (CVEP) via Michel-Penot subdifferentials, we recall some notions of generalized convexity. A real-valued function $f$ is called quasiconvex at $\bar{x}$ on $C$ if, $\forall x \in C$,

$$
f(x) \leqslant f(\bar{x}) \Longrightarrow f(\lambda x+(1-\lambda) \bar{x}) \leqslant f(\bar{x})(\forall \lambda \in(0,1)) .
$$

$f$ is said to be quasiconvex on $C$ if it is quasiconvex at each point of $C$. In case $f$ is Fréchet differentiable at $\bar{x}$, if $f$ is quasiconvex at $\bar{x}$ on $C$, then

$$
f(x)-f(\bar{x}) \leqslant 0 \Longrightarrow\langle\nabla f(\bar{x}), x-\bar{x}\rangle \leqslant 0(\forall x \in C) .
$$

The Fréchet differentiable function $f$ is said to be quasilinear at $\bar{x}$ on $C$ if $\pm f$ are quasiconvex at $\bar{x}$ on $C$.

Adapting the definition by Reiland [19], a locally Lipschitz real-valued function $f$ defined on $X$ is said to be $\partial^{M P}$-pseudoconvex at $\bar{x}$ on a subset $C$ of $X$ if

$$
\left(\exists \xi \in \partial^{M P} f(\bar{x})\right)\langle\xi, x-\bar{x}\rangle \geqslant 0 \Longrightarrow f(x)-f(\bar{x}) \geqslant 0(\forall x \in C) .
$$

In case $f$ is Fréchet differentiable at $\bar{x}, f$ is pseudoconvex at $\bar{x}$ on $C$, we have

$$
\langle\nabla f(\bar{x}), x-\bar{x}\rangle \geqslant 0 \Longrightarrow f(x)-f(\bar{x}) \geqslant 0(\forall x \in C),
$$

as $\partial^{M P} f(\bar{x})=\{\nabla f(\bar{x})\}$. The locally Lipschitz function $f$ is called $\partial^{M P}$-quasiconvex at $\bar{x}$ on $C$ if

$$
f(x)-f(\bar{x}) \leqslant 0 \Longrightarrow\langle\xi, x-\bar{x}\rangle \leqslant 0\left(\forall \xi \in \partial^{M P} f(\bar{x}), \forall x \in C\right) .
$$

Replacing $\partial^{M P}$ by $\partial^{C}$, we obtain the notions of $\partial^{C}$-quasiconvex and $\partial^{C}$-pseudoconvex functions. Note that for a locally Lipschitz real-valued function $f$ defined on $X$, one has $\partial^{M P} f(\bar{x}) \subseteq \partial^{C} f(\bar{x})$. Hence, if $f$ is $\partial^{M P}$-pseudoconvex at $\bar{x}$ on a subset $C$, then it is $\partial^{C}$-pseudoconvex at $\bar{x}$ on $C$, and if $f$ is $\partial^{C}$-quasiconvex at $\bar{x}$ on $C$, then it is $\partial^{M P}$-quasiconvex at $\bar{x}$ on $C$.

A sufficient condition for Heig efficient solutions of (CVEP) can be stated as follows.

Theorem 4.1. Let $\bar{x} \in K$. Assume that Assumption 3.7 is fulfilled. Suppose that there exist $\Lambda \in Q^{\Delta}(B), \bar{\mu}_{i} \geqslant$ $0(i \in I(\bar{x})), \bar{v}_{j} \in \mathbb{R}(j \in J)$ such that

$$
0 \in \partial^{M P}\left(\Lambda \circ F_{\bar{x}}\right)(\bar{x})+\sum_{i \in I(\bar{x})} \bar{\mu}_{i} \partial^{M P} g_{i}(\bar{x})+\sum_{j \in L} \bar{v}_{j} \nabla h_{j}(\bar{x})+N_{C}(\bar{x}) .
$$

Assume that the set $C$ is convex, the mapping $\Lambda \circ F_{\bar{x}}$ is $\partial^{M P}$-pseudoconvex at $\bar{x}$ on $C$, the mappings $g_{i}(i \in I(\bar{x}))$ are $\partial^{M P}$-quasiconvex at $\bar{x}$ on $C$, and the mappings $h_{1}, \ldots, h_{\ell}$ are quasilinear at $\bar{x}$ on $C$. Then $\bar{x}$ is a Henig efficient solution of (CVEP).

Proof. It follows from (4.1) that there exist $\xi \in \partial^{M P}\left(\Lambda \circ F_{\bar{x}}\right)(\bar{x}), \zeta_{i} \in \partial^{M P} g_{i}(\bar{x})(i \in I(\bar{x})), \eta \in N(C ; \bar{x})$ such that

$$
\xi+\sum_{i \in I(\bar{x})} \bar{\mu}_{i} \zeta_{i}+\sum_{j \in L} \bar{\gamma}_{j} \nabla h_{j}(\bar{x})+\eta=0
$$

Hence, for any $x \in C$,

$$
\langle\xi, x-\bar{x}\rangle+\sum_{i \in I(\bar{x})} \bar{\mu}_{i}\left\langle\zeta_{i}, x-\bar{x}\right\rangle+\sum_{j \in L} \bar{\gamma}_{j}\left\langle\nabla h_{j}(\bar{x}), x-\bar{x}\right\rangle+\langle\zeta, x-\bar{x}\rangle=0 .
$$


For $x \in K, g_{i}(x) \leqslant 0=g_{i}(\bar{x})(\forall i \in I(\bar{x}))$. Due to the $\partial^{M P}$-quasiconvexity of $g_{i}$ at $\bar{x}$, it holds that

$$
\left\langle\zeta_{i}, x-\bar{x}\right\rangle \leqslant 0(\forall i \in I(\bar{x})) .
$$

For $x \in K, h_{j}(x)=0=h_{j}(\bar{x})(j \in L)$. In view of the quasilinearity of $\pm h_{j}$ at $\bar{x}(j \in L)$, it results that

$$
\left.\left\langle\nabla h_{j}(\bar{x}), x-\bar{x}\right\rangle=0(j \in L)\right) .
$$

Moreover, since $C$ is convex, it follows that $T(K ; \bar{x})=\mathbb{R}_{+}(C-\bar{x})$. Hence,

$$
\langle\eta, x-\bar{x}\rangle \leqslant 0 .
$$

Substituting (4.3), (4.4) and (4.5) into (4.2) yields that

$$
\langle\xi, x-\bar{x}\rangle \geqslant 0(\forall x \in K) .
$$

By virtue of the $\partial^{M P}$-pseudoconvexity of $\Lambda \circ F_{\bar{x}}$, one gets

$$
\left(\Lambda \circ F_{\bar{x}}\right)(x) \geqslant 0(\forall x \in K) .
$$

Next, we show that $\bar{x}$ is a Henig efficient solution of (CVEP). Assume the contrary, that $\bar{x}$ is not a Henig efficient solution of (CVEP). Then, it follows from (3.3) that for every absolutely convex neighborhood $U$ of 0 with $U \subseteq V_{B}$,

$$
F(\bar{x}, K) \cap\left(-\operatorname{int} Q_{U}(B)\right) \neq \emptyset .
$$

Consequently, for every absolutely convex neighborhood $U$ of 0 with $U \subseteq V_{B}$, there exists $x_{1} \in K$ such that

$$
F_{\bar{x}}\left(x_{1}\right) \in-\operatorname{int} Q_{U}(B) .
$$

On the other hand, taking account of Proposition 3.2, there exists an absolutely convex neighborhood $U_{1}$ of 0 with $U_{1} \subseteq V_{B}$ such that $\Lambda \in Q_{U_{1}}(B)^{*} \backslash\{0\}$, as $\Lambda \in Q^{\Delta}(B)$. Hence,

$$
\left(\Lambda \circ F_{\bar{x}}\right)\left(x_{1}\right)<0,
$$

which contradicts (4.6). Hence, we obtain the desired conclusion.

On using Theorem 4.1, we get the following sufficient optimality condition for superefficient solutions of (CVEP).

Corollary 4.2. Let $\bar{x} \in K$. Assume that the base $B$ of $Q$ is bounded and closed, and all the hypotheses of Theorem 4.1 are fulfilled. Then $\bar{x}$ is a superefficient solution of (CVEP).

Proof. We invoke Theorem 4.1 to deduce that $\bar{x}$ is a Henig efficient solution of (CVEP). Since the base $B$ of $Q$ is bounded close, in view of Proposition 3.6, we deduce that $\bar{x}$ is a superefficient solution of (CVEP).

By an argument analogous to that used for the proof of Theorem 4.1, we get the following sufficient conditions for Henig solutions of (CVEP).

Theorem 4.3. Let $X=\mathbb{R}^{n}, Y=\mathbb{R}^{p}$ and $\bar{x} \in K$. Assume that Assumption 3.7 is fulfilled. Suppose that (i) There exist $\bar{\lambda} \in Q^{\Delta}(B), \bar{\mu}_{i} \geqslant 0(i \in I(\bar{x})), \bar{v}_{j} \in \mathbb{R}(j \in J)$, such that

$$
0 \in \bar{\lambda} \partial_{J} F_{\bar{x}}(\bar{x})+\sum_{i \in I(\bar{x})} \bar{\mu}_{i} \partial^{M P} g_{i}(\bar{x})+\sum_{j \in L} \bar{v}_{j} \nabla h_{j}(\bar{x})+N_{C}(\bar{x}) .
$$


(ii) The set $C$ is convex, the mapping $\bar{\lambda} \circ F_{\bar{x}}$ is $\partial^{C}$-pseudoconvex at $\bar{x}$ on $C$, the mappings $g_{i}(i \in I(\bar{x}))$ are $\partial^{M P}$-quasiconvex at $\bar{x}$ on $C$, and the mappings $h_{1}, \ldots, h_{\ell}$ are quasilinear at $\bar{x}$ on $C$.

Then $\bar{x}$ is a Henig efficient solution of (CVEP).

From Theorem 4.3, one gets the following sufficient condition for superefficient solutions of (CVEP).

Corollary 4.4. Let $X=\mathbb{R}^{n}, Y=\mathbb{R}^{p}$ and $\bar{x} \in K$. Assume that the base $B$ of $Q$ is bounded and closed, and Assumption 3.7 is fulfilled. Suppose that

(i) There exist $\bar{\lambda} \in Q^{\Delta}(B), \bar{\mu}_{i} \geqslant 0(i \in I(\bar{x})), \bar{v}_{j} \in \mathbb{R}(j \in J)$, such that

$$
0 \in \bar{\lambda} \partial_{J} F_{\bar{x}}(\bar{x})+\sum_{i \in I(\bar{x})} \bar{\mu}_{i} \partial^{M P} g_{i}(\bar{x})+\sum_{j \in L} \bar{v}_{j} \nabla h_{j}(\bar{x})+N_{C}(\bar{x}) .
$$

(ii) The set $C$ is convex, the mapping $\bar{\lambda} \circ F_{\bar{x}}$ is $\partial^{C}$-pseudoconvex at $\bar{x}$ on $C$, the mappings $g_{i}(i \in I(\bar{x}))$ are $\partial^{M P}$-quasiconvex at $\bar{x}$ on $C$, and the mappings $h_{1}, \ldots, h_{\ell}$ are quasilinear at $\bar{x}$ on $C$.

Then $\bar{x}$ is a superefficient solution of (CVEP).

Proof. The conclusion follows from Theorem 4.3 and Proposition 3.6.

In case that $F_{\bar{x}}$ is strictly differentiable, we obtain the following sufficient condition for Henig efficient solutions of (CVEP).

Theorem 4.5. Let $\bar{x} \in K$. Assume that $F_{\bar{x}}$ is strictly differentiable at $\bar{x}, g_{i}(i \in I(\bar{x}))$ are locally Lipschitz at $\bar{x}, h_{j}(j \in L)$ are Fréchet differentiable at $\bar{x}$. Suppose also that there exist $\bar{\lambda} \in Q^{\Delta}(B), \bar{\mu}_{i} \geqslant 0(i \in I(\bar{x}))$, $\bar{v}_{j} \in \mathbb{R}(j \in J)$ such that

$$
0 \in\left[D_{s} F_{\bar{x}}(\bar{x})\right]^{*} \bar{\lambda}+\sum_{i \in I(\bar{x})} \bar{\mu}_{i} \partial^{M P} g_{i}(\bar{x})+\sum_{j \in L} \bar{v}_{j} \nabla h_{j}(\bar{x})+N_{C}(\bar{x}) .
$$

Suppose, in addition, that the set $C$ is convex, the mapping $\bar{\lambda} \circ F_{\bar{x}}$ is pseudoconvex at $\bar{x}$ on $C$, the mappings $g_{i}(i \in I(\bar{x}))$ are $\partial^{M P}$-quasiconvex at $\bar{x}$ on $C$, and the mappings $h_{1}, \ldots, h_{\ell}$ are quasilinear at $\bar{x}$ on $C$. Then $\bar{x}$ is a Henig efficient solution of (CVEP).

From Theorem 4.5, we get the following sufficient condition for superefficient solutions of (CVEP).

Corollary 4.6. Let $\bar{x} \in K$. Assume that $F_{\bar{x}}$ is strictly differentiable at $\bar{x}$, the base $B$ of $Q$ is bounded and closed, and all the hypotheses of Theorem 4.5 are fulfilled. Then $\bar{x}$ is a superefficient solution of (CVEP).

Proof. The conclusion follows from Theorem 4.5 and Proposition 3.6.

Remark 4.7. If the base $B$ of $Q$ is bounded closed, then the condition $\bar{\lambda} \in Q^{\Delta}(B)$ in Theorems 4.3, 4.5 can be replaced by $\bar{\lambda} \in$ int $Q^{*}$.

\section{APPLICATIONS TO VECTOR VARIATIONAL INEQUALITY AND OPTIMIZATION PROBLEMS}

This section will be devoted to describing some applications of the results obtained in Sections 3 and 4 to vector variational inequalities and vector optimization problems.

Let $L(X ; Y)$ be the space of all continuous linear mappings from $X$ to $Y$, and let $T$ be a mapping from $X$ to $L(X ; Y)$. The vector equilibrium problem (CVEP) includes as a special case the constrained vector variational inequality (CVVI) with $F(x, y)=T(x)(y-x)$, where $X, Y, F, K$ are as in Section 3. A vector $\bar{x} \in K$ is a local Henig solution or a local superefficient solution of (CVEP) with $F(\bar{x}, y)=T(\bar{x})(y-\bar{x})$ 
will be called a local Henig efficient solution or local superefficient solution of (CVVI), respectively. If $F(x, y)=f(y)-f(x)(x, y \in X)$, where $f: X \rightarrow Y$, then the vector equilibrium problem (CVEP) becomes the following constrained vector optimization problem (CVOP):

$$
\min \{f(x): x \in K\} \text {. }
$$

A vector $\bar{x} \in K$ is a local Henig solution or a local superefficient solution of (CVEP) with $F(\bar{x}, y)=$ $f(y)-f(\bar{x})$ will be called a local Henig efficient solution or local superefficient solution of (CVOP), respectively.

A necessary optimality condition for local Henig efficient solution and local superefficient solution of the constrained vector variational inequality (CVVI) can be stated as follows.

Theorem 5.1. Let $\bar{x}$ be a local Henig efficient solution or local superefficient solution of (CVVI). Assume that $H(\bar{x})$ is weakly* closed, Assumption 3.7 and Constraint qualification $(C Q)$ hold. Then there exist $\bar{\lambda} \in Q^{\Delta}(B), \bar{\mu}_{i} \geqslant 0(i \in I(\bar{x})), \bar{v}_{j} \in \mathbb{R}(j \in J)$ such that

$$
0 \in[T(\bar{x})]^{*} \bar{\lambda}+\sum_{i \in I(\bar{x})} \bar{\mu}_{i} \partial^{M P} g_{i}(\bar{x})+\sum_{j \in L} \bar{v}_{j} \nabla h_{j}(\bar{x})+N_{C}(\bar{x}) .
$$

Moreover, if the base B of $Q$ is bounded closed, then $\bar{\lambda} \in$ int $Q^{*}$, where int $Q^{*}$ is the interior of $Q^{*}$ with respect to the strong topology on $Y^{*}$.

Proof. (a) $\bar{x}$ is a local Henig efficient solution: It can be seen that $F_{\bar{x}}(\bar{x})=0$. Since $T(\bar{x})$ is a continuous linear mapping from $X$ to $Y$, it is strictly differentiable. Taking account of Theorem 3.12, we obtain the derised conclusion.

(b) $\bar{x}$ is a local superefficient solution: Making use of Proposition 3.6 and Part (a), one gets the required conclusion immediately.

A Kuhn-Tucker necessary condition for Henig efficiency and superefficiency of (CVOP) can be formulated as follows.

Theorem 5.2. Let $\bar{x}$ be a local Henig efficient solution or local superefficient solution of (CVOP). Assume that $H(\bar{x})$ is weakly* closed, Constraint qualification $(C Q)$ and Assumption 3.7, in which $F_{\bar{x}}$ is replaced by $f$, hold. Then

(i) There exist $\bar{\lambda} \in Q^{\Delta}(B), \bar{\mu}_{i} \geqslant 0(i \in I(\bar{x})), \bar{v}_{j} \in \mathbb{R}(j \in J)$, and a continuous positively homogeneous function $\Lambda$ on $Y$ satisfying $(\alpha),(\beta)$ such that

$$
0 \in \partial^{M P}(\Lambda \circ f)(\bar{x})+\sum_{i \in I(\bar{x})} \bar{\mu}_{i} \partial^{M P} g_{i}(\bar{x})+\sum_{j \in L} \bar{v}_{j} \nabla h_{j}(\bar{x})+N_{C}(\bar{x})
$$

(ii) If, in addition, $f$ is strictly differentiable at $\bar{x}$, then there exist $\bar{\lambda} \in Q^{\Delta}(B), \bar{\mu}_{i} \geqslant 0(i \in I(\bar{x})), \bar{v}_{j} \in$ $\mathbb{R}(j \in J)$ such that

$$
0 \in\left[D_{s} f(\bar{x})\right]^{*} \bar{\lambda}+\sum_{i \in I(\bar{x})} \bar{\mu}_{i} \partial^{M P} g_{i}(\bar{x})+\sum_{j \in L} \bar{v}_{j} \nabla h_{j}(\bar{x})+N_{C}(\bar{x})
$$

Moreover, if the base B of $Q$ is bounded closed, then $\bar{\lambda} \in$ int $Q^{*}$, where int $Q^{*}$ is the interior of $Q^{*}$ with respect to the strong topology on $Y^{*}$. 
(iii) Let $X=\mathbb{R}^{n}, Y=\mathbb{R}^{p}$. Assume that all the hypotheses of Theorem 3.8 hold. Then there exist $\bar{\lambda} \in Q^{\Delta}(B)$, $\bar{\mu}_{i} \geqslant 0(i \in I(\bar{x})), \bar{v}_{j} \in \mathbb{R}(j \in J)$, such that

$$
0 \in \bar{\lambda} \partial_{J} F_{\bar{x}}(\bar{x})+\sum_{i \in I(\bar{x})} \bar{\mu}_{i} \partial^{M P} g_{i}(\bar{x})+\sum_{j \in L} \bar{v}_{j} \nabla h_{j}(\bar{x})+N_{C}(\bar{x}) .
$$

Moreover, if the base $B$ of $Q$ is bounded closed, then $\bar{\lambda} \in$ int $Q^{*}$.

Proof. (i) If $\bar{x}$ is a local Henig efficient solution of (CVOP), invoking Theorem 3.8 and observing that

$$
\partial^{M P}(f-f(\bar{x}))(\bar{x})=\partial^{M P} f(\bar{x})
$$

we then arrive the conclusion (i). If $\bar{x}$ is a local superefficient solution of (CVOP), taking account of Proposition 3.6, we also arrive the desired conclusion.

(ii) If $\bar{x}$ is a local Henig efficient solution of (CVOP), on using Theorem 3.12, we then obtain the required conclusion. If $\bar{x}$ is a local superefficient solution of (CVOP), in view of Proposition 3.6, we arrive the desired conclusion.

(iii) Making use of Theorem 3.10 and Proposition 3.6 we also get the desired conclusion immediately.

A sufficient optimality condition for (CVVI) can be stated as follows.

Theorem 5.3. Let $\bar{x} \in K$, and $Q$ have a base B. Assume that there exist $\bar{\lambda} \in Q^{\Delta}(B), \bar{\mu}_{i} \geqslant 0(i \in I(\bar{x}))$, $\bar{v}_{j} \in \mathbb{R}(j \in J)$ such that

$$
0 \in[T(\bar{x})]^{*} \bar{\lambda}+\sum_{i \in I(\bar{x})} \bar{\mu}_{i} \partial^{M P} g_{i}(\bar{x})+\sum_{j \in L} \bar{v}_{j} \nabla h_{j}(\bar{x})+N_{C}(\bar{x}) .
$$

Suppose, in addition, that the set $C$ is convex, the mappings $g_{i}(i \in I(\bar{x}))$ are $\partial^{M P}$-quasiconvex at $\bar{x}$ on $C$, and the mappings $h_{1}, \ldots, h_{\ell}$ are quasilinear at $\bar{x}$ on $C$. Then $\bar{x}$ is a Henig efficient solution of $(C V V I)$.

Proof. Since $T(\bar{x})$ is a continuous linear mapping from $X$ to $Y$, it is strictly differentiable. For $F_{\bar{x}}(y)=$ $T(\bar{x})(y-\bar{x})$, one has $D_{s} F_{\bar{x}}(\bar{x})=T(\bar{x})$. For $\bar{\lambda} \in Q^{\Delta}(B)$, the mapping $\bar{\lambda} \circ T(\bar{x})$ is linear, and hence, pseudoconvex. Consequently, we can invoke Theorem 4.5 for (CVVI) to deduce the desired conclusion.

A sufficient condition for superefficiency of (CVVI) can be formulated as follows.

Corollary 5.4. Let $\bar{x} \in K$. Assume the base $B$ of $Q$ is bounded closed, and all the hypotheses of Theorem 5.3 are fulfilled in which $\bar{\lambda} \in Q^{\Delta}(B)$ is replaced by $\bar{\lambda} \in$ int $Q^{*}$, where int $Q^{*}$ is the interior of $Q^{*}$ with respect to the strong topology on $Y^{*}$. Then $\bar{x}$ is a superefficient solution of (CVVI).

Proof. Applying Theorem 5.3 yields that $\bar{x}$ is a Henig efficient solution of (CVVI). Taking account of Proposition 3.6, we obtain the required conclusion.

The following sufficient condition is a direct consequence of Theorem 4.1 to (CVOP).

Theorem 5.5. Let $\bar{x} \in K$, and $Q$ have a base B. Suppose that there exist $\Lambda \in Q^{\Delta}(B), \bar{\mu}_{i} \geqslant 0(i \in I(\bar{x}))$, $\bar{v}_{j} \in \mathbb{R}(j \in J)$ such that

$$
0 \in \partial^{M P}(\Lambda \circ f)(\bar{x})+\sum_{i \in I(\bar{x})} \bar{\mu}_{i} \partial^{M P} g_{i}(\bar{x})+\sum_{j \in L} \bar{v}_{j} \nabla h_{j}(\bar{x})+N_{C}(\bar{x}) .
$$


Assume that the set $C$ is convex, the mapping $\Lambda \circ f$ is $\partial^{M P}$-pseudoconvex at $\bar{x}$ on $C$, the mappings $g_{j}(j \in$ $I(\bar{x})$ are $\partial^{M P}$-quasiconvex at $\bar{x}$ on $C$, and the mappings $h_{1}, \ldots, h_{\ell}$ are quasilinear at $\bar{x}$ on $C$. Then $\bar{x}$ is a Henig efficient solution of (CVOP).

Proof. Applying Theorem 4.1, we get that $\bar{x}$ is a Henig efficient solution of (CVEP) with $F(\bar{x}, y)=$ $f(y)-f(\bar{x})$. Hence, the desired conclusion follows.

In case $f$ is strictly differentiable, we obtain the following sufficient condition for Henig efficient solutions.

Theorem 5.6. Let $\bar{x} \in K$, and $Q$ have a base B. Assume that $f$ is strictly differentiable at $\bar{x}$. Suppose, in addition, that there exist $\bar{\lambda} \in Q^{\Delta}(B), \bar{\mu}_{i} \geqslant 0(i \in I(\bar{x})), \bar{v}_{j} \in \mathbb{R}(j \in J)$ such that

$$
0 \in\left[D_{s} f(\bar{x})\right]^{*} \bar{\lambda}+\sum_{i \in I(\bar{x})} \bar{\mu}_{i} \partial^{M P} g_{i}(\bar{x})+\sum_{j \in L} \bar{v}_{j} \nabla h_{j}(\bar{x})+N_{C}(\bar{x}) .
$$

Suppose that the set $C$ is convex, the function $\bar{\lambda} \circ f$ is pseudoconvex at $\bar{x}$ on $C$, the mappings $g_{j}(j \in I(\bar{x})$ are $\partial^{M P}$-quasiconvex at $\bar{x}$ on $C$, and the mappings $h_{1}, \ldots, h_{\ell}$ are quasilinear at $\bar{x}$ on $C$. Then $\bar{x}$ is a Henig efficient solution of (CVOP).

Proof. Applying Theorem 4.5 to function $F(x, y)=f(y)-f(x)$, we get the desired conclusion immediately.

In case the base $B$ is bounded closed, we can state the following sufficient condition for superefficiency of (CVOP).

Corollary 5.7. Let $\bar{x} \in K$. Assume the base $B$ of $Q$ is bounded closed, and all the hypotheses of Theorem 5.6 are fulfilled in which $\bar{\lambda} \in Q^{\Delta}(B)$ is replaced by $\bar{\lambda} \in$ int $Q^{*}$, where int $Q^{*}$ is the interior of $Q^{*}$ with respect to the strong topology on $Y^{*}$. Then $\bar{x}$ is a superefficient solution of (CVOP).

Proof. Making use of Theorem 5.6, we get that $\bar{x}$ is a Henig solution of (CVOP). Due to Proposition 3.6, $\bar{x}$ is a superefficient solution for (CVOP).

Theorem 5.8. Let $X=\mathbb{R}^{n}, Y=\mathbb{R}^{p}$ and $\bar{x} \in K$. Assume that the base $B$ of $Q$ is bounded closed, and Assumption 3.7 is fulfilled. Suppose that

(i) There exist $\bar{\lambda} \in Q^{\Delta}(B), \bar{\mu}_{i} \geqslant 0(i \in I(\bar{x})), \bar{v}_{j} \in \mathbb{R}(j \in J)$, such that

$$
0 \in \bar{\lambda} \partial_{J} f(\bar{x})+\sum_{i \in I(\bar{x})} \bar{\mu}_{i} \partial^{M P} g_{i}(\bar{x})+\sum_{j \in L} \bar{v}_{j} \nabla h_{j}(\bar{x})+N_{C}(\bar{x})
$$

(ii) The set $C$ is convex, the mapping $\bar{\lambda} \circ f$ is $\partial^{C}$-pseudoconvex at $\bar{x}$ on $C$, the mappings $g_{i}(i \in I(\bar{x}))$ are $\partial^{M P}$-quasiconvex at $\bar{x}$ on $C$, and the mappings $h_{1}, \ldots, h_{\ell}$ are quasilinear at $\bar{x}$ on $C$.

Then $\bar{x}$ is a Henig efficient solution of (CVOP). Moreover, if the base $B$ of $Q$ is bounded and closed, then $\bar{x}$ is a superefficient solution of $(C V O P)$.

Proof. Using Theorem 4.3 and Proposition 3.6, we find the desired conclusion immediately. 


\section{CONCLUSiOnS}

Based on a Kuhn-Tucker necessary condition due to Luu [14] and a scalarization result by Gong [3], we derive Kuhn-Tucker necessary conditions for local Henig efficient solutions and local superefficient solutions of vector equilibrium problems involving equality, inequality and set constraints with locally Lipschitz functions under the constraint qualification of Abadie type via the Michel-Penot subdifferentials. Sufficient optimality conditions for Henig efficiency and superefficiency can be established under some assumptions on generalized convexity. It can be seen that making use of results on necessary optimality conditions for nonsmooth multiobjective optimization problems in terms of the Michel-Penot subdifferentials, we can derive necessary optimality conditions for efficiency of nonsmooth constrained vector equilibrium problems. The Kuhn-Tucker necessary conditions obtained here via the MichelPenot subdifferentials can be sharper than those expressed in terms of the Clarke subdifferentials. The results obtained in this paper are more general than those obtained by Gong [3] for vector equilibrium problems with only a set constraint, and those obtained by Long-Huang-Peng [8] for vector equilibrium problems with subconvexlike functions.

\section{Acknowledgements}

The authors would like to thank the referees for their valuable comments and suggestions which improve the paper. This research was funded by Vietnam National Foundation for Science and Technology Development (NAFOSTED) under Grant number 101.01-2017.301.

\section{REFERENCES}

[1] P. Daniele, Lagrange multipliers and infinite-dimensional equilibrium problems, J. Glob. Optim. 40 (2008), 65-70.

[2] F. Giannessi, G. Mastroeni, L. Pellegrini, On the theory of vector optimization and variational inequalities, image space analysis and separation, in: Vector Variational Inequalities and Vector Equilibria: Mathematical Theories, F. Giannessi (ed.), Kluwer, Dordrecht, pp. 153-215, (2000).

[3] X.H. Gong, Scalarization and optimality conditions for vector equilibrium problems, Nonlinear Anal. 73 (2010), 3598 3612.

[4] X.H. Gong, W.T. Fu, W. Liu, Superefficiency for vector equilibrium in locally convex topological vector spaces, in: Vector Variational Inequalities and Vector Equilibria: Mathematical Theories, F. Giannessi (ed.), Kluwer, Dordrecht, pp. 233-252, (2000)

[5] I.V. Girsanov, Lectures on Mathematical Theory of Extremum Problems, Berlin-Heidenberg, Springer-Verlag, 1972.

[6] T.X.D. Ha, Optimality conditions for various efficient solutions involving coderivatives: From set-valued optimization problems to set-valued equilibrium problems, Nonlinear Anal. 75 (2012), 1305-1323.

[7] P.Q. Khanh, L.T. Tung, First and second-order optimality conditions using approximations for vector equilibrium problems with constraints, J. Glob. Optim. 55 (2013), 901-920.

[8] X.J. Long, Y.Q. Huang, Z.Y. Peng, Optimality conditions for the Henig efficient solution of vector equilibrium problems with constraints, Optim. Lett. 5 (2011), 717-728.

[9] J. Morgan, M. Romaniello, Scalarization and Kuhn-Tucker-like conditions for weak vector generalized quasivariational inequalities, J. Optim. Theory Appl. 130 (2006), 309-316.

[10] D.V. Luu, D.D. Hang, On optimality conditions for vector variational inequalities, J. Math. Anal. Appl. 412 (2014), 792-804.

[11] D.V. Luu, D.D. Hang, Efficient solutions and optimality conditions for vector equilibrium problems, Math. Meth. Oper. Res. 79 (2014), 163-177.

[12] D.V. Luu, D.D. Hang, On efficiency conditions for nonsmooth vector equilibrium problems with equilibrium constraints, Numer. Funct. Anal. Optim. 36 (2015), 1622-1642. 
[13] X. Qin, J.C. Yao, Projection splitting algorithms for nonself operators, J. Nonlinear Convex Anal. 18 (2017), 925-935.

[14] D.V. Luu, Necessary conditions for efficiency in terms of the Michel-Penot subdifferentials, Optimization 61 (2012), 1099-1117.

[15] P. Michel, J.-P. Penot, Calcul sous-différentiel pour des fonctions lipschitziennes et nonlipschitziennes, C. R. Acad. Sci. Paris Sér. I Math. 12 (1984), 269-272.

[16] F. H. Clarke, Optimization and Nonsmooth Analysis, Wiley Interscience, New York, 1983.

[17] W. Schirotzek, Nonsmooth Analysis, Springer, Berlin Heidelberg New York, 2007.

[18] V. Jeyakumar, D.T. Luc, Nonsmooth calculus, minimality, and monotonicity of convexificators, J. Optim. Theory Appl. 101 (1999), 599-621.

[19] T.W. Reiland, A geometric approach to nonsmooth optimization with sample applications, Nonlinear Anal. 11 (1987), 1169-1184. 